\title{
Producción y rendimiento reproductivo en Petrolisthes granulosus (Decapoda, Anomura, Porcellanidae) en diferentes localidades del norte de Chile: Una comparación latitudinal
}

\author{
Patricio Hernáez Bové \\ Universidad Arturo Prat, Av. 11 de Septiembre 2120, Casilla 121, Iquique, Chile \\ E-mail: patobove@eudoramail.com
}

Recibido: 19 julio 1999; versión corregida: 19 julio 2000; aceptado: 21 marzo 2001

\begin{abstract}
RESUMEN. Se colectaron hembras ovígeras del cangrejo porcelánido Petrolisthes granulosus para analizar las variaciones latitudinales de la biología reproductiva en la zona norte de Chile. Las muestras se obtuvieron en cinco localidades distribuidas entre los $18^{\circ} 33^{\prime} \mathrm{S}$ y $25^{\circ} 20^{\prime} \mathrm{S}$. Los valores promedios de fecundidad, volumen del huevo en la etapa final del desarrollo embrionario, rendimiento reproductivo y talla de mínima madurez sexual mostraron cierta tendencia latitudinal. Aunque estos resultados corroboran de alguna manera el efecto de la latitud sobre la biología reproductiva de P. granulosus, contradicen lo descrito por otros autores (Thorson, 1936, 1950; Clarke, 1987, 1992) con respecto al comportamiento reproductivo latitudinal de otras especies marinas.
\end{abstract}

Palabras claves: latitud, biología reproductiva, Petrolisthes, Chile.

\section{Production and reproductive output in Petrolisthes granulosus (Decapoda, Anomura, Porcellanidae) in different localities in the northern zone of Chile: A comparison latitudinal}

\begin{abstract}
Ovigerous females of the porcellanid crab Petrolisthes granulosus were collected in the northern zone of Chile, to analyze latitudinal variations in their reproductive biology. Samples were taken from five localities situated between $18^{\circ} 33^{\prime} \mathrm{S}$ to $25^{\circ} 20^{\prime} \mathrm{S}$. The average fecundity values for both locality and size strata, size of minimum sexual maturity, reproductive output and egg volume in the final stage of embryonic development, showed to have a tendency to the increase with the latitude. Though this result corroborate the latitudinal effect on the reproductive biology of $P$. granulosus, on the other hand, they contradict Thorson's $(1936,1950)$ and Clarke's $(1987,1992)$ arguments regarding latitudinal reproductive behavior.
\end{abstract}

Key words: latitude, reproductive biology, Petrolisthes, Chile.

\section{INTRODUCCIÓN}

La variación latitudinal en la ecología reproductiva de una misma especie constituye información relevante que permite conocer las adaptaciones al ambiente (Sastry, 1970). En el pasado, varios autores han propuesto y discutido la respuesta reproductiva frente al cambio latitudinal (Thorson, 1936, 1950; Clarke, 1987, 1992; Gorny et al., 1992). Se ha establecido que la temperatura es el principal factor regulador del crecimiento y rendimiento reproductivo en muchos organismos marinos (Giese, 1959; Sastry, 1966; Jones y Simons, 1983), por lo cual toda variación en los patrones reproductivos de una espe- cie distribuida en una amplia zona geográfica, estarán asociados a un gradiente térmico latitudinal (Clarke 1987; 1992).

En Chile, diversos autores han reportado importantes diferencias latitudinales en el número y volumen de los huevos producidos por porcelánidos y camarones carideos de las zonas norte y sur (Lardies y Wehrtmann, 1996; López et al., 1997; Wehrtmann y Kattner, 1998). Sin embargo, estos antecedentes parecen insuficientes y no permiten corroborar la supuesta influencia de la latitud sobre las características reproductivas de crustáceos decápodos. 
Petrolisthes granulosus (Guerín, 1835), es un pequeño cangrejo que habita bajo rocas intermareales en todo el borde costero de la zona norte de Chile y que se distribuye desde Paita, Perú, a Coronel, Chile (Haig, 1960; Retamal, 1981). En el presente estudio, se discute y analiza la respuesta reproductiva de $P$. granulosus frente al cambio latitudinal, sobre la base de antecedentes descritos anteriormente en literatura, como una forma de contribuir al conocimiento autoecológico de esta especie en el norte de Chile.

\section{MATERIALES Y MÉTODOS}

Se colectaron hembras ovígeras de Petrolisthes granulosus desde cinco localidades ubicadas en la zona comprendida entre los $18^{\circ} 33^{\prime} \mathrm{S}$ y $25^{\circ} 20^{\prime} \mathrm{S}$ (Fig. 1). Las muestras fueron obtenidas entre el 4 y 14 de noviembre de 1997, época en que esta especie alcanza su máximo reproductivo anual (Sanhueza $e t$ al., 1975). El período de captura se redujo al mínimo para evitar cambios temporales en las características reproductivas de cada población (Díaz, 1980; Jones, 1980). En cada localidad se capturaron manualmente, gran cantidad de individuos (durante marea de sicigia), tratando de abarcar toda la zona de distribución de esta especie en la franja intermareal. Luego se separaron arbitrariamente las hembras ovígeras del resto de los individuos, se almacenaron en cajas con hielo y transportaron rápidamente al Departamento de Química de la Universidad Arturo Prat, donde fueron congeladas a una temperatura de $-15^{\circ} \mathrm{C}$ para su posterior análisis.

Una vez en laboratorio, a cada hembra se le midió la longitud del cefalotórax (Lc) desde el ápice del rostro a la región posterior del caparazón y el ancho o amplitud del caparazón (Ac) desde los márgenes laterales del cefalotórax; todas estas mediciones fueron realizadas utilizando un vernier de 0,02 $\mathrm{mm}$ de precisión. Para determinar el número de huevos portados por cada hembra (Nth), se separó la masa ovígera bajo lupa estereoscópica, y se contaron y clasificaron según su estado de desarrollo. El estado de desarrollo en los huevos (Ed) fue dividido en tres según el siguiente criterio (Wehrtmann, 1990): Estado I: huevos recientemente producidos, vitelo uniforme, sin mancha ocular visible. Estado II: mancha ocular escasamente visible. Estado III: ojos visibles y ampliamente pigmentados, abdomen libre.

Para estimar el volumen del huevo ( $\mathrm{Vh})$, se se- pararon arbitrariamente 20 unidades por hembra a las que se midió la longitud y ancho con un microscopio con ocular graduado; estos datos fueron incorporados a la fórmula $\mathrm{Vh}=1 / 6\left(\left(\mathrm{~b}^{2} * \mathrm{a}\right) * \pi\right)$ (Corey y Reid, 1991). La inversión energética en el proceso reproductivo fue estimada por medio del rendimiento reproductivo (Ro) de los huevos en estado inicial de desarrollo; para ello las hembras y sus masas ovígeras fueron desecadas a una temperatura de $60^{\circ} \mathrm{C}$ por un tiempo de $24 \mathrm{hr}$, una vez completado este proceso se enfriaron a temperatura ambiente y se pesaron en una balanza analítica digital marca Sartorius de $10^{-4} \mathrm{~g}$ de precisión; posteriormente estos resultados se incorporaron a la fórmula propuesta por Clarke et al. (1991) para el cálculo del Ro.

Se confeccionaron diagramas de dispersión para la relación Nth v/s Lc y Ac. Posteriormente, cada relación fue sometida a análisis de regresión para determinar la ecuación que explique de mejor manera la producción de huevos en cada localidad. Para

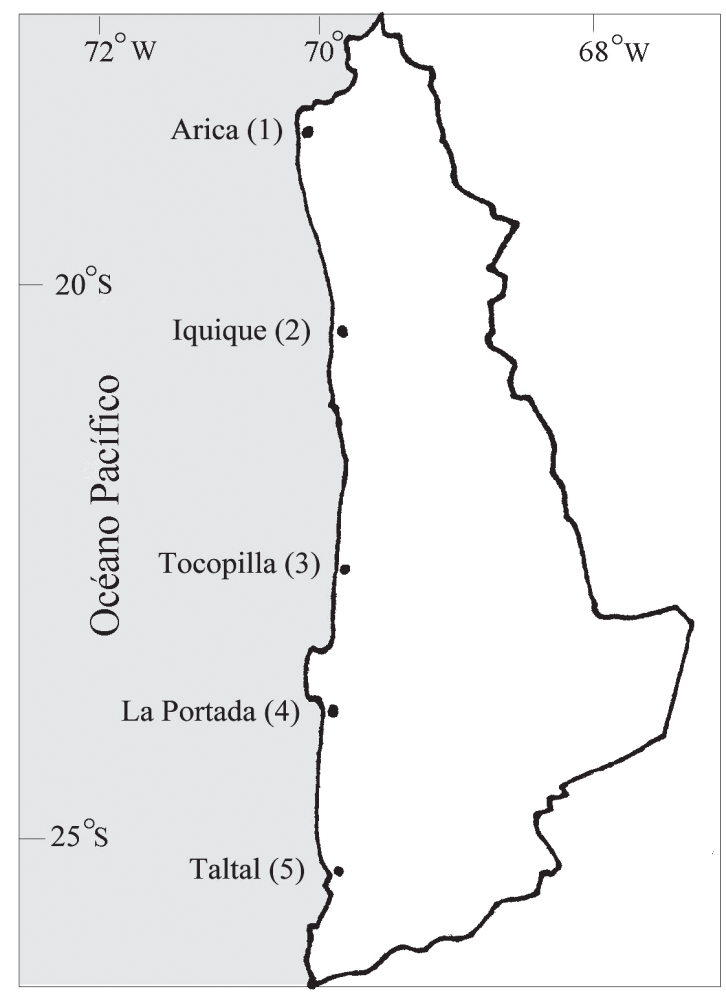

Figura 1. Localidades de recolección de las hembras de Petrolisthes granulosus (Localidades 1-5), zona norte de Chile.

Figure 1. Collection localities for females of Petrolisthes granulosus (Localities 1-5), in northern Chile. 
establecer la relación entre la latitud y cada una de las variables reproductivas medidas, se aplicaron modelos de regresión lineal (método de mínimos cuadrados) entre las medias de la longitud del caparazón (Lc), fecundidad (Nth), volumen de cada estado del desarrollo (Vh), rendimiento reproductivo (Ro) y cada localidad.

Por último, las medias de cada variable reproductiva se sometieron a análisis de varianza y se confrontaron entre sí con el test de Tukey $(\mathrm{P}<$ $0,05)$, para establecer posibles diferencias entre localidades.

\section{RESULTADOS}

Durante el período de estudio se analizaron 483 hembras ovígeras de Petrolisthes granulosus, que fluctuaron entre 4,3 y $11,6 \mathrm{~mm}$ de longitud cefalotorácica (Lc), y produjeron entre 15 y 516 huevos durante la incubación. Se encontraron diferencias significativas $(\mathrm{P}<0,05)$ entre cada una de las variables reproductivas analizadas a lo largo de toda la zona de estudio (Tabla 1).

Tomando la longitud del caparazón como una aproximación a la madurez sexual de las hembras (Giese, 1959; Jones, 1980; Simons y Jones, 1981), la talla de maduración de $P$. granulosus aumentó progresivamente desde Arica (4,3 mm Lc) a Tocopilla (5,7 mm Lc), luego disminuyó levemente en las localidades 4 y 5, donde las hembras alcanzan su pubertad sobre los 5,3 mm de longitud cefalotorácica. El valor medio del largo del caparazón (Lc) encontrado en cada localidad aumentó considerablemente con respecto a la localidad de menor latitud geográfica (Arica), algo similar se encontró en la producción promedio por localidad, la cual no presentó diferencias significativas $(\mathrm{P}>0,05)$ entre las localidades 4 y 5 (Tabla 1).

La producción de huevos aumentó considerablemente con la talla de las hembras. En la mayoría de los casos, el número de huevos producidos se relacionó fuertemente con el ancho del caparazón (Tabla 2). La distribución de frecuencia del número de huevos en clases de tamaño (longitud del caparazón), indica la correlación positiva entre estas variables por cada localidad y cuando estas fueron agrupadas (Tabla 3 y Fig 2, respectivamente). En general, la función que mejor se ajusta a la variable número de huevos (Nth) es la del modelo de poder $\left(\mathrm{y}=\mathrm{a} * \mathrm{x}^{\mathrm{b}}\right)$ y el ajuste a esta función en cada variable corporal se indica en la Figura 3.

Durante el período de incubación, el volumen del huevo fluctuó entre 0,139-0,171 m³3 0,160$0,176 \mathrm{~mm}^{3}$ y $0,177-0,217 \mathrm{~mm}^{3}$ en los estados de desarrollo I, II y III respectivamente. En cada localidad, el huevo presentó un importante aumento de volumen hacia el final de la embriogénesis; este incremento fue mayor en aquellas poblaciones distri-

Tabla 1. Petrolisthes granulosus: Rangos y promedios de la longitud cefalotoráxica (Lc), número de huevos producidos (Nth) y volumen del huevo (Vh) de las hembras presentes en diferentes localidades del norte de Chile. Desviación estándar (SD) y número de individuos analizados (n).

Table 1. Petrolisthes granulosus: Mean and range values for carapace length (Lc), egg number (Nth) and mean volume of individual eggs (Vh) for females in different localities off northern Chile. Standard deviation (SD) and number of individuals analysed.

\begin{tabular}{|c|c|c|c|c|c|c|c|c|c|c|}
\hline \multirow[b]{2}{*}{ Localidad } & \multirow[b]{2}{*}{$\mathbf{n}$} & \multicolumn{3}{|c|}{$\begin{array}{l}\text { Longitud del caparazón } \\
(\mathrm{mm})\end{array}$} & \multicolumn{3}{|c|}{$\begin{array}{c}\text { Número de huevos } \\
\text { (Nth) }\end{array}$} & \multicolumn{3}{|c|}{$\begin{array}{c}\text { Volumen del huevo } \\
\left(\mathrm{mm}^{3}\right)\end{array}$} \\
\hline & & Rango & Media & SD & Rango & Media & SD & Rango & Media & SD \\
\hline 1 & 118 & $4,3-10,5$ & $6,8 \mathrm{a}$ & 1,3 & $15-380$ & $88 \mathrm{a}$ & 54 & $0,116-0,240$ & $0,169 \mathrm{a}$ & 0,021 \\
\hline 2 & 84 & $4,6-11,4$ & $7,8 \mathrm{~b}$ & 1,5 & $23-324$ & $114 \mathrm{~b}$ & 67 & $0,124-0,243$ & $0,185 \mathrm{~b}$ & 0,022 \\
\hline 3 & 92 & $5,7-11,6$ & $8,9 \mathrm{c}$ & 1,4 & $42-516$ & $210 \mathrm{c}$ & 86 & $0,126-0,251$ & $0,175 \mathrm{ab}$ & 0,027 \\
\hline 4 & 93 & $5,5-10,3$ & $7,8 \mathrm{~b}$ & 1,2 & $39-356$ & $152 \mathrm{~d}$ & 71 & $0,113-0,311$ & $0,177 \mathrm{ab}$ & 0,046 \\
\hline 5 & 96 & $5,3-11,1$ & $8,3 \mathrm{~d}$ & 1,5 & $35-393$ & $171 \mathrm{~d}$ & 85 & $0,116-0,252$ & $0,179 \mathrm{ab}$ & 0,032 \\
\hline $\mathrm{F}$ & & \multicolumn{3}{|c|}{32,2} & \multicolumn{3}{|c|}{43,0} & \multicolumn{3}{|c|}{3,6} \\
\hline $\mathrm{P}$ & & \multicolumn{3}{|c|}{$<0,001$} & \multicolumn{3}{|c|}{$<0,001$} & \multicolumn{3}{|c|}{$<0,01$} \\
\hline
\end{tabular}

Las medias seguidas por una misma letra en la columna no difieren estadísticamente entre sí $(\mathrm{P}>0,05)$. 
Tabla 2. Petrolisthes granulosus: Funciones para la estimación de la producción de huevos en diferentes localidades del norte de Chile. Coeficiente de determinación $\left(\mathbf{r}^{2}\right)$.

Table 2. Petrolisthes granulosus: Functions for egg product estimation from different localities off northern Chile. Coefficient of determination $\left(\mathbf{r}^{2}\right)$.

\begin{tabular}{|c|c|c|c|c|c|}
\hline Localidad & $\mathbf{x}$ & $\mathbf{n}$ & Función & $\mathbf{r}^{2}(\%)$ & $\mathbf{F}$ \\
\hline \multirow[t]{2}{*}{1} & $\mathrm{Lc}$ & 118 & $\mathrm{Nth}=1,69 * \mathrm{Lc}^{2,00}$ & 44,8 & $94,1^{*}$ \\
\hline & $\mathrm{Ac}$ & 118 & $\mathrm{Nth}=1,03^{*} \mathrm{Ac}^{2,38}$ & 52,5 & $128,1 *$ \\
\hline \multirow[t]{2}{*}{2} & $\mathrm{Lc}$ & 84 & $\mathrm{Nth}=0,59 * \mathrm{Lc}^{2,50}$ & 62,7 & $138,0^{*}$ \\
\hline & $\mathrm{Ac}$ & 84 & $\mathrm{Nth}=0,53 * \mathrm{Ac}^{2,76}$ & 67,4 & $169,1^{*}$ \\
\hline \multirow[t]{2}{*}{3} & $\mathrm{Lc}$ & 92 & $\mathrm{Nth}=1,89 * \mathrm{Lc}^{2,12}$ & 54,8 & $109,1 *$ \\
\hline & $\mathrm{Ac}$ & 92 & $\mathrm{Nth}=1,84 * \mathrm{Ac}^{2,25}$ & 64,2 & $161,0 *$ \\
\hline \multirow[t]{2}{*}{4} & $\mathrm{Lc}$ & 93 & $\mathrm{Nth}=0,66 * \mathrm{Lc}^{2,60}$ & 69,7 & $209,5^{*}$ \\
\hline & $\mathrm{Ac}$ & 93 & $\mathrm{Nth}=0,72 * \mathrm{Ac}^{2,72}$ & 73,8 & $256,3^{*}$ \\
\hline \multirow[t]{2}{*}{5} & $\mathrm{Lc}$ & 96 & $\mathrm{Nth}=0,78 * \mathrm{Lc}^{2,50}$ & 74,6 & $275,7 *$ \\
\hline & $\mathrm{Ac}$ & 96 & $\mathrm{Nth}=1,09 * \mathrm{Ac}^{2,48}$ & 75,6 & $290,9 *$ \\
\hline
\end{tabular}

* Todos significantes a un nivel de $\mathrm{P}<0,001$. buidas más al sur de la zona de estudio (Tabla 4). Las hembras provenientes de la localidad 1 producen en promedio huevos de menor volumen que el resto de las poblaciones estudiadas (Tabla 1).

La inversión energética en el proceso reproductivo expresada como rendimiento reproductivo (Ro), presentó una importante disminución hacia las localidades 1 y 2 . En toda la zona de estudio las hembras de $P$. granulosus invirtieron en el proceso reproductivo entre un $3,75 \%$ a $6,84 \%$ de su peso seco (Fig. 4).

Tabla 3. Petrolisthes granulosus: Distribución del número de huevos (media \pm desviación estándar) por clase de tamaño (Lc) en cada una de las localidades estudiadas.

Table 3. Petrolisthes granulosus: Distribution of the number of eggs (mean \pm standar deviation) for size class (Lc) in each localities.

\begin{tabular}{|c|ccccc|c|}
\hline \multirow{2}{*}{$\begin{array}{c}\text { Clase } \\
\text { de Lc } \\
(\mathbf{m m})\end{array}$} & Arica (1) & Iquique (2) & Tocopilla (3) & La Portada (4) & Taltal (5) & \multirow{2}{*}{ Total } \\
\cline { 2 - 6 } & $36 \pm 18(11)^{*}$ & $34 \pm 11(3)$ & - & - & - & $36 \pm 1(14)$ \\
$4-5$ & $59 \pm 23(29)$ & $51 \pm 25(4)$ & $51 \pm 13(2)$ & $78 \pm 23(9)$ & $57 \pm 13(10)$ & $61 \pm 8(54)$ \\
$5-6$ & $82 \pm 25(25)$ & $64 \pm 23(24)$ & $104 \pm 18(5)$ & $84 \pm 17(17)$ & $99 \pm 23(14)$ & $81 \pm 13(85)$ \\
$6-7$ & $101 \pm 32(34)$ & $111 \pm 34(23)$ & $148 \pm 44(22)$ & $127 \pm 34(25)$ & $127 \pm 37(17)$ & $120 \pm 17(121)$ \\
$7-8$ & $125 \pm 66(13)$ & $151 \pm 66(10)$ & $202 \pm 63(23)$ & $191 \pm 50(28)$ & $186 \pm 43(21)$ & $179 \pm 26(95)$ \\
$8-9$ & $190 \pm 121(5)$ & $159 \pm 67(10)$ & $279 \pm 75(20)$ & $217 \pm 36(8)$ & $224 \pm 65(23)$ & $227 \pm 41(66)$ \\
$9-10$ & $268 \pm 0(1)$ & $206 \pm 65(9)$ & $260 \pm 77(14)$ & $291 \pm 56(6)$ & $285 \pm 79(9)$ & $258 \pm 31(39)$ \\
$10-11$ & - & $208 \pm 0(1)$ & $261 \pm 61(6)$ & - & $326 \pm 95(2)$ & $270 \pm 36(9)$ \\
$11-12$ & & &
\end{tabular}

*Número de hembras utilizadas en el cálculo de la media por clase de tamaño.

Tabla 4. Petrolisthes granulosus: Volumen promedio del huevo en cinco localidades del norte de Chile. Incremento en el volumen (\%) durante la incubación (Ed = I-III).

Table 4. Petrolisthes granulosus: Mean volume $\left(\mathrm{mm}^{3}\right)$ of the egg during the embryonic development. Increment of volumen (\%) between stages I and III (Ed).

\begin{tabular}{|c|c|c|c|c|c|c|c|}
\hline \multirow[b]{3}{*}{ Localidad } & \multicolumn{6}{|c|}{ Volumen del huevo $\left(\mathrm{mm}^{3}\right)$} & \multirow{3}{*}{ I-III $(\%)$} \\
\hline & \multicolumn{2}{|c|}{ E-I } & \multicolumn{2}{|c|}{ E-II } & \multicolumn{2}{|c|}{ E-III } & \\
\hline & $\mathbf{n}$ & Media & $\mathbf{N}$ & Media & n & Media & \\
\hline 1 & 40 & $0,164 \mathrm{a}$ & 24 & $0,160 \mathrm{a}$ & 52 & 0,177 a & 7,9 \\
\hline 2 & 27 & $0,171 \mathrm{~b}$ & 17 & $0,176 \mathrm{a}$ & 40 & $0,198 \mathrm{~b}$ & 15,8 \\
\hline 3 & 39 & $0,159 \mathrm{ab}$ & 18 & $0,160 \mathrm{a}$ & 35 & $0,199 \mathrm{~b}$ & 25,2 \\
\hline 4 & 30 & $0,139 \mathrm{c}$ & 30 & $0,171 \mathrm{a}$ & 33 & $0,217 \mathrm{c}$ & 56,1 \\
\hline 5 & 36 & $0,160 \mathrm{ab}$ & 26 & $0,173 \mathrm{a}$ & 34 & $0,205 \mathrm{bc}$ & 28,1 \\
\hline
\end{tabular}

Las medias seguidas por una misma letra en la columna no difieren estadísticamente entre sí $(\mathrm{P}>0,05)$. 


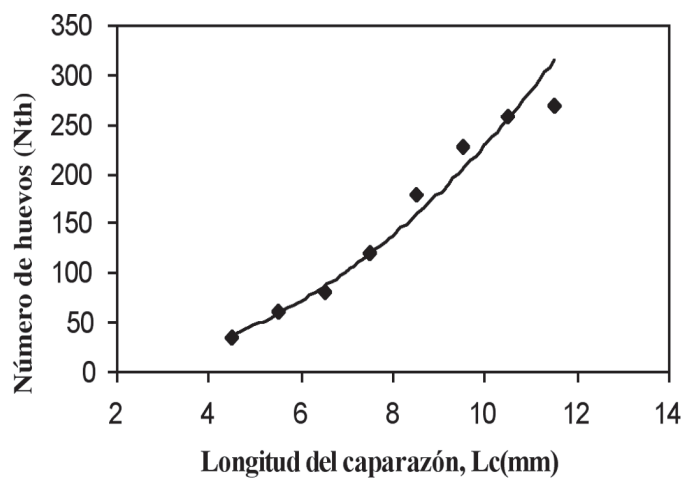

Figura 2. Petrolisthes granulosus: Ajuste a la función de poder a de la media del número de huevos por clase de tamaño (longitud del caparazón), para el número total de hembras colectadas en diferentes localidades del norte de Chile.

Figure 2. Petrolisthes granulosus: Mean value of the number of eggs fitted to the potential function for every size class (length of carapace), for the total number of females collected in northern Chile.
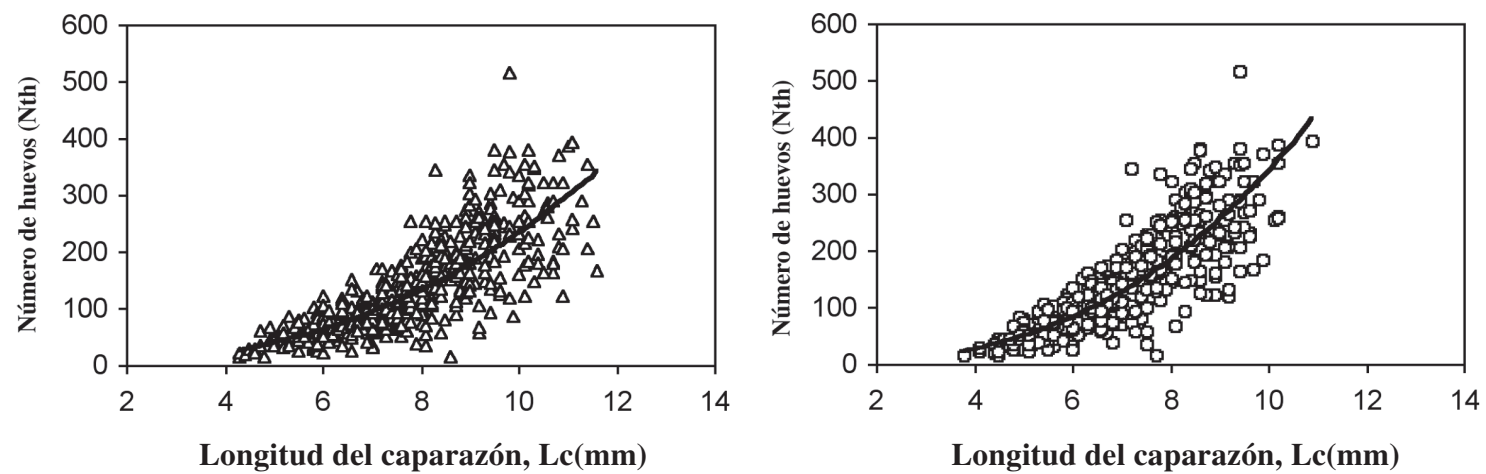

Figura 3. Petrolisthes granulosus: Ajuste a la función de poder a la relación tamaño corporal (Lc; Ac) y la producción de huevos, para todas las hembras analizadas en las localidades de estudio, en el norte de Chile.

Figure 3. Petrolisthes granulosus: Fitted potential function for the relationship between body size (Lc; Ac) and egg production for the total number of females collected in northern Chile.

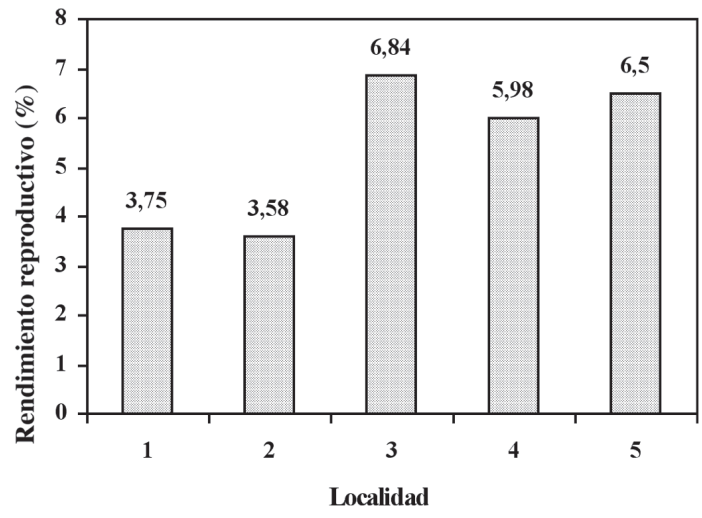

Figura 4. Petrolisthes granulosus: Rendimiento reproductivo de las hembras en diferentes localidades del norte de Chile.

Figure 4. Petrolisthes granulosus: Output reproductive of females in different localities in the northern zone of Chile.

\section{DISCUSIÓN}

La biología reproductiva de $P$. granulosus presentó una clara influencia latitudinal (Tabla 5), los antecedentes registrados en el presente trabajo contradicen las tendencias descritas por algunos autores con respecto al comportamiento latitudinal de las especies marinas.

\section{Tamaño corporal y producción de huevos}

El tamaño de los individuos es una de las variables mejor documentadas en literatura (Vernberg, 1962; Kinne, 1970; Hasting, 1981; Clarke, 1992). Se sabe que el tamaño corporal y la talla de madurez sexual de los organismos aumentan progresivamente con la latitud; esta tendencia a menudo es relacionada con la temperatura y las diferencias metabólicas que se producen a lo largo del rango latitudinal de una especie, es decir, a bajas temperaturas los individuos crecen y maduran más lentamente que a temperaturas mayores (Annala et al., 1980; Clarke et al., 1991). Lo encontrado en $P$. granulosus confir- 
Tabla 5. Petrolisthes granulosus: Análisis de regresión para cada uno de los parámetros reproductivos y la latitud entre poblaciones $(\mathbf{n}=\mathbf{5})$.

Table 5. Petrolisthes granulosus: Regression analysis for each of the reproductive parameters and latitude between populations $(\mathbf{n}=\mathbf{5})$.

\begin{tabular}{|lccc|}
\hline Parámetro reproductivo & r & F & P \\
\hline Lc & 0,60 & 1,66 & 0,29 \\
Talla mínima (Lc) & 0,73 & 3,53 & 0,16 \\
Fecundidad (Nth) & 0,65 & 2,19 & 0,24 \\
Volumen total (Vh) & 0,35 & 0,41 & 0,57 \\
Volumen (E-I) & 0,51 & 1,04 & 0,38 \\
Volumen (E-II) & 0,47 & 0,86 & 0,42 \\
Volumen (E-III) & 0,81 & 5,70 & 0,10 \\
Ro & 0,78 & 4,70 & 0,12 \\
\hline
\end{tabular}

Tabla 6. Petrolisthes granulosus: Análisis de regresión entre parámetros reproductivos en la zona de estudio $(\mathbf{n}=5)$.

Table 6. Petrolisthes granulosus: Regression analysis for reproductive parameters in the study zone $(n=5)$.

\begin{tabular}{|ccccc|}
\hline $\mathrm{x}$ & $\mathrm{y}$ & $\mathrm{r}$ & $\mathrm{F}$ & $\mathrm{P}$ \\
\hline Lc & Nth & 0,95 & 27,57 & 0,01 \\
Lc & Ro & 0,80 & 5,25 & 0,11 \\
Ro & Nth & 0,94 & 20,89 & 0,02 \\
\hline
\end{tabular}

ma en cierta manera dicha proposición, ya que la talla de maduración sexual y el tamaño medio de las hembras se correlacionan positivamente con la latitud (Tabla 5). Sin embargo, la ausencia de registros térmicos durante el período de estudio impiden confirmar esta relación.

La estrategia que las hembras de crustáceos decápodos adoptan durante el período reproductivo es el resultado de la interacción de factores de orden endógeno y exógeno (Pinheiro y Fransozo, 1995). Las variaciones locales de estos factores generan importantes diferencias en el número y tamaño de los huevos producidos por las hembras (Jones y Simons, 1983); en este sentido, diversos autores han postulado la existencia de una disminución en la producción hacia altas latitudes debido al incremento en el volumen del huevo (Thorson,1936, 1950; Clarke, 1979; Clarke et al., 1991; Gorny et al., 1992). Los resultados obtenidos, contradicen parcialmente esta proposición, ya que las hembras tienden a producir más huevos en las localidades ubicadas a mayor latitud dentro de la zona de estudio. La fecundidad registrada entre Tocopilla y Taltal presentó un relativo descenso, a pesar de ello, la producción en estas localidades es mayor a la registrada entre Arica e Iquique (Tabla 1).

Los huevos de porcelánidos son relativamente ovoides y crecen considerablemente durante el desarrollo hasta alcanzar tamaños que van desde 13,6\% a $120 \%$ de su volumen inicial (Petrolisthes haigae y $P$. armatus, respectivamente). Esto genera una disminución en la fecundidad producto de la falta de espacio disponible (Reid y Corey, 1991), es decir, el área bajo el abdomen limitará físicamente el número de huevos producidos. Esta proposición permite plantear la hipótesis que en Petrolisthes granulosus la longitud del caparazón y la producción aumentan progresivamente con la latitud. Esta hipótesis parece verdadera, ya que latitudinalmente se observó una fuerte correlación entre el tamaño medio de las hembras y la fecundidad de cada localidad (Tabla 6).

A pesar de algunas excepciones, gran parte de los crustáceos presentan una correlación positiva entre el número de huevos y una determinada variable corporal (Sastry, 1983); en este sentido, $P$. granulosus no es una excepción, la producción de huevos registrada en cada localidad se ve incrementada con el tamaño en las hembras (Tabla 3). Comparado con otros representantes de esta familia, $P$. granulosus produce pocos huevos, el número de huevos en esta especie se encuentra dentro del rango descrito por Antezana et al. (1965) en Liopetrolisthes mitra $(\mathrm{Nth}=65-543)$ y por Hernáez y Pinheiro (en preparación) en Petrolisthes violaceus $(\mathrm{Nth}=16-325)$. La fecundidad promedio de todas las hembras analizadas (144 huevos) es menor a la registrada por Lardies y Wehrtmann (1996) en 
Petrolisthes laevigatus (364 huevos) de la zona centro-sur de Chile, aun cuando este último alcanza un tamaño medio mayor que $P$. granulosus.

\section{Volumen del huevo y rendimiento reproductivo}

En muchos invertebrados marinos se ha observado la tendencia a producir huevos de mayor volumen a altas latitudes (Thorson, 1936, 1950; Efford, 1969; Jones y Simons, 1983). En este sentido, Clarke (1992) señala que las diferencias en el volumen del huevo detectadas en poblaciones de distintas áreas, constituyen la respuesta reproductiva de las hembras al cambio medio ambiental. El volumen del huevo registrado en cada localidad, fue similar durante casi todo el período de incubación (Tabla 4) y en general, el tamaño del huevo mostró una baja correlación con la latitud (Tabla 5). Estos resultados contradicen lo observado en diversos crustáceos decápodos (Clarke, 1979; Clarke et al., 1991; Gorny et al., 1992) donde se ha detectado un aumento en el tamaño del huevo hacia altas latitudes.

Steele y Steele (1975) señalan que la variación en el tamaño del huevo está relacionada con la duración del desarrollo embrionario y con el tipo de larva que eclosiona, es decir, huevos de gran tamaño provenientes de altas latitudes son usualmente asociados con estados avanzados de desarrollo larval y también con un incremento en el tiempo de desarrollo (Clarke, 1982). Dentro de la zona estudiada, el tiempo de incubación en los embriones aumentó considerablemente con la latitud (Tabla 4), a pesar de ello, no existe ninguna relación entre la latitud y el volumen del huevo registrado en cada localidad (Tabla 1). Lardies y Whertmann (1996) señalan que el crecimiento de los embriones en Petrolisthes laevigatus es limitado por el grosor y resistencia de la membrana del huevo, si esta afirmación es correcta, las especies sometidas a mayor presión de desecación poseerán membranas mucho más gruesas, la cual impedirá la pérdida de agua y limitará el crecimiento acelerado de sus embriones. Los resultados encontrados por Hernáez y Pinheiro (en preparación) en Allopetrolisthes angulosus 48,0\% y A. spinifrons $51,0 \%$, confirman la propuesta anterior, ya que estas especies se encuentran sometidas a menor presión de desecación que $P$. granulosus por ubicarse más abajo en la zona intermareal. Comparado con otros miembros de la familia, . granulosus produce huevos relativamente grandes, el volumen del huevo en este cangrejo es mayor al observado en $A$. angulosus $\left(0,089 \mathrm{~mm}^{3}\right)$ y A. spinifrons $(0,119$ $\mathrm{mm}^{3}$ ), aun cuando estas dos especies alcanzan un tamaño medio mayor.

$P$ granulosus presentó un rendimiento gónadopeso específico (Ro) similar al de P. laevigatus $5,57 \%$ (Lardies y Wehrtmann, 1996), pero muy por debajo al registrado en camarones carideos de regiones templadas y polares, cuyos promedios fluctúan entre los $11,8 \%$ en Notocrangon antarcticus y 24,2\% en Pandalus montagui (Clarke, 1987; Clarke et al., 1991). La información descrita en camarones pandálidos e hippolytidos sugiere que la inversión reproductiva de las especies marinas disminuye hacia las zonas polares (Clarke, 1987). Los valores encontrados no siguen este esquema, el rendimiento reproductivo de $P$. granulosus muestra cierta tendencia a aumentar con la latitud (Fig. 4). Al parecer el incremento en la producción detectado en las localidades de mayor ubicación latitudinal, causa un mayor gasto de energía en el transporte de los huevos por parte de las hembras (Clarke, 1979); esto último se demuestra al correlacionar ambas variable en el plano latitudinal (Tabla 6). Esta última observación va en desmedro de lo registrado en otros crustáceos marinos, donde se ha reportado una disminución en la producción y rendimiento reproductivo con el incremento en latitud (Lonsdale y Levington, 1985; Jones y Simons, 1983; Clarke, 1987; Clarke y Gore, 1992; Gorny et al., 1992).

Aun cuando lo observado en $P$. granulosus contradice las principales propuestas reproductivas frente al cambio latitudinal (Thorson, 1936, 1950; Clarke, 1987, 1992), se requiere efectuar otros estudios que contemplen un mayor esfuerzo para comprobar esta tendencia.

\section{AGRADECIMIENTOS}

Vayan mis más sinceros agradecimientos a los colegas Sr. Guillermo Guzmán G. por los buenos consejos a la hora de preparar el texto y a la Prof. Fresia Bravo P. por su desinteresada ayuda en terreno, ambos fueron fundamentales en la concreción de este documento.

\section{REFERENCIAS}

Annala, J.H., J.L. Mckoy, J.D. Booth y R.B. Pike. 1980. Size at the onzet of sexual maturity in female Jasus edwarsii (Decapoda: Palinuridae) in New Zeland. N. Z. J. Mar. Freshwat. Res., 14: 217-228. 
Antezana, T., E. Fagetti y M.T. López. 1965. Observaciones bioecológicas en decápodos de Valparaíso. Rev. Biol. Mar., 12: 1-60.

Clarke, A. 1979. On living in cold water: K-strategies in Antarctic benthos. Mar. Biol., 55: 111-119.

Clarke, A. 1982. Temperature and embryonic development in polar marines invertebrates. Int. J. Invert. Repr., 5: 71-82.

Clarke, A. 1987. Temperature, latitude and reproductive effort. Mar. Ecol. Prog. Ser., 38: 89-99.

Clarke, A., C.C. Hopkins y E.M. Nilssen. 1991. Egg size and reproductive output in the deepwater pranw Pandalus borealis Kroyer, 1838. Funct. Ecol., 5: 724-730.

Clarke, A. 1992. Reproduction in the cold: Thorson revisited. Invertebr. Reprod. Dev., 22(1-3): 175184.

Clarke, A. y D.J. Gore. 1992. Egg size and composition Ceratoselis (Crustacea: Isopoda) from the Weddell Sea. Polar Biol., 12: 129-134.

Corey, S. y D.M. Reid. 1991. Comparative fecundity of decapod crustaceans. I. The fecundity of thirty tree species of nine families of caridean shrimps. Crustaceana, 60(3): 270-294.

Diaz, H. 1980. The mole crab Emerita talpoida (Say): a case of changing life history pattern. Ecol. Monogr., 50: 437-456.

Efford, I.E. 1969. Egg size in the sand crab. Emerita analoga (Decapoda, Hippidae). Crustaceana, 16: 15-26.

Giese, A.C. 1959. Comparative phisyology: annual reproductive cycles of marine invertebrates. Annu. Rev. Physiol., 21: 547-576.

Gorny, M., W.E. Arntz, A. Clarke y D.J. Gore. 1992. Reproductive biology of caridean decapods from the Weddell Sea. Polar Biol., 12: 111-120.

Haig, J. 1960. The Porcellanidae (Crustacea, Anomura) of the Eastern Pacific. Allan Hancock Pacific Expedition, 24: 1-440.

Hasting, M.H. 1981. The life cycle and productivity of and intertidal population of the amphipod Ampelisca brevicornis. Estuar. Coast. Shelf Sci., 12: 665-677.
Jones, M.B. 1980. Reproductive ecology of the estuarine burrowing mud crab Helice crassa (Grapsidae). Estuar. Coast. Shelf. Sci., 11: 433-443.

Jones, M.B. y M.J. Simons. 1983. Latitudinal variation in reproductive characteristics of a mud crab, Helice crassa (Grapsidae). Bull. Mar. Sci., 33: 656-670.

Kinne, O. 1970. Temperature: animals-invertebrates. En: O. Kinne (ed.). Marine ecology. I. Environmental factors. Wiley-Interscience. London, pp. 407-514.

Lardies, M.A. y I.S. Wehrtmann. 1996. Aspects of the reproductive biology of Petrolisthes laevigatus (Guerin, 1835) (Decapoda, Anomura, Porcellanidae). Part I: Reproductive output and chemical composition of eggs during embryonic development. Arch. Fish. Mar. Res., 43(2): 121135.

López, L., T. Jeri, C. González y S. Rodríguez. 1997. Fecundidad y esfuerzo reproductivo de Petrolisthes granulosus (Guerín, 1835) en Iquique, Chile (Decapoda, Anomura, Porcellanidae). Invest. Mar., Valparaíso, 25: 159-166.

Lonsdale, D.J. y J.S. Levington. 1985. Latitudinal differentiation in embryonic duration, egg size, and newborn survival in a harpacticoid copepod. Biol. Bull., 168: 419-431.

Pinheiro, M.A. y A. Fransozo. 1995. Fecundidade de Pachycheles haigae Rodrigues da Costa, 1960 (Crustacea, Anomura, Porcellanidae) em Ubatuba (SP), Brasil. Rev. Brasil. Biol., 55(4): 623-631.

Reid, D.M. y S. Corey. 1991. Comparative fecundity of decapod crustaceans. II. The fecundity of fifteen species of anomuran and brachyuran crabs. Crustaceana, 61(2): 175-189.

Retamal, M.A. 1981. Catálogo ilustrado de los crustáceos decápodos de Chile. Gayana, Zool., 44: 1110.

Sanhueza, E., N. Bahamonde y M.T. López. 1975. Petrolisthes granulosus (Guérin) en biocenosis supramareales de El Tabo (Crustacea, Decapoda, Anomura). Bol. Mus. Nac. Hist. Nat., 34: 121-136.

Sastry, A.N. 1966. Temperature effects in reproduction of the bay scallop, Aequipecten irradians Lamarck. Biol. Bull., 130: 118-134. 
Sastry, A.N. 1970. Reproductive physiological variation in latitudinally separated populations of the bay scallop, Aequipecten irradians Lamarck. Biol. Bull., 138: 56-65.

Sastry, A.N. 1983. Ecological aspects of reproduction. En: F.J. Vernberg and W.B. Vernberg (eds.). The Biology of Crustacea. Environmental adaptations. New York, Academic Press, Inc., pp. 179-270.

Simons, M.J. y M.B. Jones. 1981. Population and reproductive biology of the mud crab, Macrophthalmus hirtipes (Jacquinot, 1853) (Ocypodidae), from marine estuarine habitats. J. Nat. Hist., 15: 981-994.

Steele, D.H. y V.J. Steele. 1975. Egg size and duration embryonic development in Crustacea. Int. Rev. ges. Hydrobiol., 60(5): 711-715.
Thorson, G. 1936. The larval development, growth and metabolism of Arctic marine invertebrates, compared with those of the other seas. Meddr. Gronland., 100: 1-155.

Thorson, G. 1950. Reproductive and larval ecology of marine bottom invertebrates. Biol. Rev., 25: 145 .

Vernberg, F.J. 1962. Latitudinal effects on physiological properties of animal populations. Annu. Rev. Physiol., 24: 517-546.

Wehrtmann, I.S. 1990. Distribution and reproduction of Ambidexter panamense and Palaemonetes schmitii in Pacific Costa Rica (Crustacea, Decapoda). Rev. Biol. Trop., 38(2A): 327-329.

Wehrtmann, I.S. y G. Kattner. 1998. Changes in volume, biomass and fatty acids of developing eggs in Nauticaris magellanica (Decapoda: Caridea): A latitudinal comparison. J. Crust. Biol., 18(3): 413422 . 
